\title{
Réduction des interférences locales lors de l'usinage 5 axes sur le flanc de surfaces complexes : utilisation de fraise tonneau
}

\author{
Julien Chaves-Jacob ${ }^{1, a}$, Richard Chatain ${ }^{2}$ et Gérard Poulachon ${ }^{1}$ \\ 1 LaBoMaP, ENSAM de Cluny, 71250 Cluny, France \\ 2 Mécachrome, Bureau Technique, 27-49 Ave. Eugène Cassela, 18700 Aubigny, France
}

Reçu le 15 mars 2007, accepté le 6 juillet 2007

\begin{abstract}
Résumé - De nos jours l'usinage de surfaces complexes reste une opération délicate à maîtriser. Deux procédés permettent d'obtenir de telles surfaces en usinage : le fraisage en roulant avec une fraise cylindrique ou conique et le fraisage en bout avec une fraise hémisphérique ou torique. Le fraisage en roulant étant plus productif que le fraisage en bout, il serait donc intéressant de l'utiliser pour usiner tout type de surface. Le problème de ce mode de génération de surface est qu'il engendre des interférences locales (positives ou négatives) par rapport à la surface théorique. La solution innovante proposée par ces travaux consiste à jouer sur la forme de l'outil en utilisant des fraises à géométrie particulière en forme de tonneau, le but étant de diminuer les interférences locales. L'objectif de cette étude est la géométrie de la fraise tonneau en fonction de la surface et des tolérances imposées.
\end{abstract}

Mots clés : Usinage 5 axes / fraisage flanc / interférences / fraise tonneau / surface complexe

Abstract - Reduction of local interferences during flank milling of free form surfaces in 5 axis machining: development of barrel cutter. Nowadays machining of free form surface is still difficult to master. Two techniques enable to machine them: flank milling with a cylindrical cutter or conical tool and point milling with a ball end milling cutter or torus cutter. Flank milling is more productive. This technique induces cutting interferences (overcut or undercut) as regards to the theoretical surface. The novel method proposed by these research works consists of using particular tool geometry such as barrel form. This intermediary solution between cylindrical and ball-end milling cutters enables to respect much more the theoretical surface in a significant reduced cutting time. The aim of this study will permit to determine the suitable barrel geometry of the milling cutter as a function of the machining surface and the imposed tolerances.

Key words: 5 axis machining / flank milling / interferences / barrel cutter / free form surface

\section{Introduction}

Les travaux qui sont présentés permettent d'apporter de nouvelles solutions aux problématiques rencontrées lors de l'usinage 5 axes en roulant. Ce type d'usinage est utilisé dans les secteurs de la pétrochimie, l'aéronautique, l'aérospatial, l'horlogerie, la bijouterie...

L'usinage de surfaces complexes reste encore de nos jours un problème délicat à maîtriser. Actuellement, 2 stratégies permettent d'usiner ces formes [1] : le fraisage en roulant avec une fraise cylindrique ou conique

\footnotetext{
a Auteur pour correspondance :

et le fraisage en bout avec une fraise hémisphérique ou torique.

Dans le cas de l'usinage de surfaces quelconques, la seule stratégie industrielle permettant d'usiner la surface avec des tolérances acceptables est le fraisage en bout d'outil.

Dans le cas de Surfaces Réglées Développables (SRD), le fraisage en roulant est, sous réserve d'accessibilité et de vibration, le procédé le plus économique.

En revanche, dans le cas des Surfaces Réglées Non Développables (SRND), l'usinage en flanc génère des interférences locales [2]. Il faut alors vérifier que les valeurs maximales de ces interférences ne gênent pas la fonction technique de la pièce. 


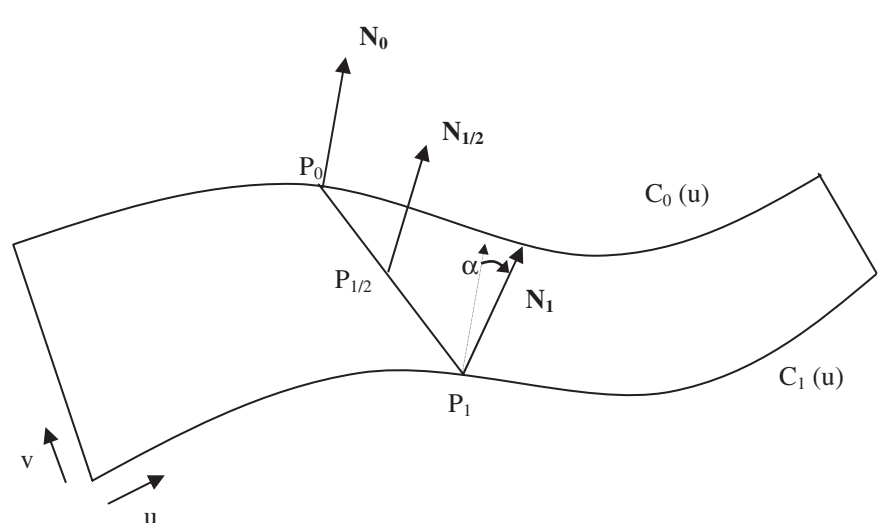

Fig. 1. Définition d'une surface réglée.

Le principal avantage de l'usinage en bout est qu'il permet d'usiner sans interférences la surface nominale. Ses deux principaux défauts sont un temps d'usinage plus grand et la formation de crêtes sur la pièce entre chaque passe. Dans certains cas, il est nécessaire de polir la surface pour faire disparaître ces crêtes.

Sur les turbomachines, les interférences trop importantes entraînent des pertes de rendement des turbines, qui peuvent être non négligeables.

L'objectif de cette étude est de déterminer la géométrie optimale de la fraise en fonction de la surface à usiner et des tolérances imposées sur cette surface

\section{Positionnement du problème}

\subsection{Surfaces réglées}

Définition : une surface réglée est une surface qui a été obtenue par balayage d'une droite, nommée règle. Cette droite est guidée par deux courbes, $C_{0}(u)$ et $C_{1}(u)$, nommées directrices. La figure 1 permet de voir les deux directrices $C_{0}$ et $C_{1}$, ainsi qu'une règle particulière $\left[P_{0} P_{1}\right]$.

Ces surfaces sont largement répandues dans l'industrie que ce soit pour modéliser des surfaces fonctionnelles ou des surfaces esthétiques. Ce type de description de surface est très utilisé dans les domaines aéronautique, spatial, naval ou automobile.

L'équation (1) d'une telle surface est donnée par :

$\mathrm{S}(u, v)=(1-v) \cdot C_{0}(u)+v \cdot C_{1}(u) \quad$ i $\quad \forall(u, v) \in[0,1]^{2}$

Notation employée :

$\left[P_{0} P_{1}\right]$ est la règle considérée;

$C_{0}(u)$ et $C_{1}(u)$ sont les deux directrices de la surface;

$\boldsymbol{N}_{0}$ et $\boldsymbol{N}_{1}$ sont les normales à la surface aux extrémités de la règle;

$\alpha=\operatorname{angle}\left(\boldsymbol{N}_{0}, \boldsymbol{N}_{1}\right)$ est la vrille de la surface pour la règle considérée.

Surfaces Réglées Développables (SRD) : les SRD sont une sous-famille des surfaces réglées. Une SRD est une surface réglée qui a une courbure gaussienne nulle en tout point. Ceci a pour conséquence que la vrille $\alpha$ est nulle pour toutes les positions de la règle. Une SRD a donc la
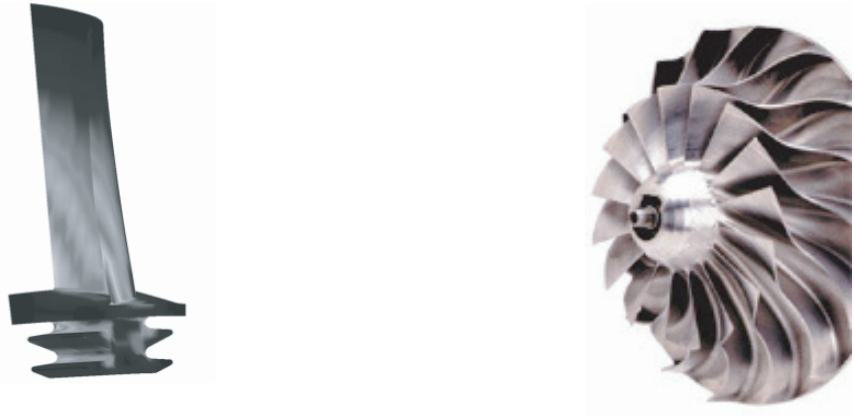

Fig. 2. Aubage de turbine et impeller [3].
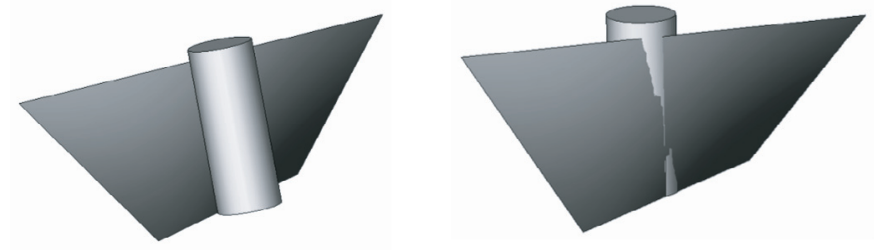

Fig. 3. Visualisation des interférences locales.

même normale le long de ses règles (exemple : cylindres, cône, plan...).

Surfaces Réglées Non Développables (SRND) : les SRND sont donc toutes les surfaces réglées qui n'ont pas la propriété d'être développables. Une conséquence est donc que les SRND ont une vrille non nulle en tout point. La figure 2 donne un exemple de pièce industrielle utilisant ce type de surface.

Dans le but de classifier les différentes SRND en famille deux indicateurs ont été créés :

- la variation de la hauteur des règles notée : $\Delta h$ est donnée par l'équation (2)

$$
\Delta h=\frac{\max (h)}{\min (h)}
$$

- la variation de la vrille de la surface : $\Delta \alpha$ est donnée par l'équation (3)

$$
\Delta \alpha=\frac{\max (\alpha)}{\min (\alpha)}
$$

Ces indicateurs permettent de quantifier la variation d'une surface. Cette variation est pénalisante pour l'emploi de fraises tonneau avec une stratégie mono-passe. En effet les fraises tonneau étant calculées de façon globale sur une surface, elles seront peu adaptées si cette surface varie beaucoup.

\subsection{Interférences d'usinage}

La figure 3 présente une interférence locale entre une SRND et une fraise cylindrique. En effet dans la vue de dessous de la surface, l'outil traverse la surface et va donc lors de l'usinage enlever trop de matière; c'est de l'overcut. À l'inverse l'outil peut aussi lors de l'usinage laisser trop de matière sur la surface c'est de l'undercut. 
Le fraisage en bout (travail d'enveloppe) permet d'obtenir la surface théorique sans interférences, mais ce procédé est moins économique et il peut nécessiter une phase de finition manuelle par polissage pour éliminer les crêtes [4].

Le fraisage en flanc (travail de forme) avec une fraise cylindrique ou conique est plus rapide et plus rentable. Son inconvénient majeur est qu'il ne peut être utilisé que sur des surfaces réglées. De plus si la surface réglée est non développable des interférences locales apparaîtront [2]. Dans la littérature de nombreuses solutions existent pour modifier le posage outil (et donc la trajectoire) de façon à réduire l'interférence générée [5-9].

\section{Méthodes de positionnement}

La programmation FAO 5 axes d'une pièce est réalisée dans l'espace pièce. Dans cet espace pour donner une position outil par rapport à la pièce il faut spécifier un point de l'outil (en général le bout d'outil) et la direction orientée de l'axe outil. L'ensemble de ces posages outil constitue la trajectoire. Pour chacun des posages la trajectoire est donc constituée des trois coordonnées, $X, Y$ et $Z$ du point de l'outil dans le repère pièce et de $I, J$ et $K$ les trois projections du vecteur directeur de l'outil dans le repère pièce. En effet, en usinage 5 axes continus, le vecteur directeur n'est jamais constant.

Il existe plusieurs méthodes pour calculer un posage outil sur une SRND [5-9]. Ces méthodes peuvent être classifiées de deux façons différentes :

- selon l'orientation de l'axe outil par rapport à la règle : s'ils ont la même orientation ou non; l'outil

- le nombre de points de tangence entre la surface et

Ici c'est la deuxième solution qui est présentée.

\subsection{Posage avec un point de tangence}

\section{Positionnement logiciel}

Ce positionnement est présent dans de nombreux logiciels de FAO. Il consiste à positionner l'axe de l'outil parallèle à la règle considérée $\left(\left[P_{0} P_{1}\right]\right.$ et l'outil tangent à une des deux directrices au point considéré $\left(P_{0}\right.$ ou $\left.P_{1}\right)$

Dans le cas où $C_{0}(u)$ est choisie comme courbe de tangence, un point de l'axe outil, $P_{\text {out }}$ est donné par l'équation (4):

$$
P_{\text {out }}=P_{0}+R \cdot N_{0}(u)
$$

avec $R$ le rayon de l'outil

L'axe de l'outil est donné par la direction de la règle.

Le choix d'être tangent à une directrice n'est pas anodin, ce choix entraîne une interférence maximale, de type overcut, sur l'autre directrice comme illustré à la figure 4 .

Avantages :

- le calcul du posage outil est très simple et permet ainsi un temps de calcul très rapide ce qui explique que

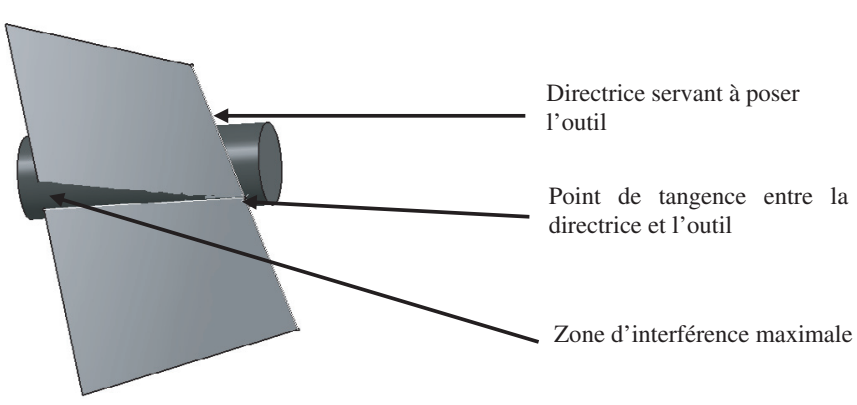

Fig. 4. Profil d'interférence obtenue avec le positionnement logiciel.

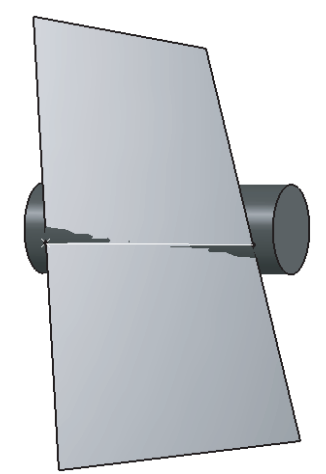

Fig. 5. Interférences avec le posage SPO.

cette solution est couramment utilisée dans les logiciels de FAO;

- l'usinage d'une des deux directrices est exécuté sans erreur.

Inconvénients :

- l'erreur d'interférence est très importante, ce qui oblige pour respecter la tolérance, à avoir des rayons d'outil petits et donc peu productif et aussi une flexion d'outil importante;

- l'erreur d'interférence n'est que de l'overcut.

\section{Positionnement SPO}

Liu [5] propose deux posages différents le SPO et le DPO. Le premier nommé Single Point Offset (SPO) consiste à faire tangenter l'outil au milieu de la règle considérée (le point $P_{1 / 2}$ sur la Fig. 1) et l'axe outil à la direction de la règle. L'équation (5) permet de calculer un point de l'outil :

$$
P_{\text {out }}=P_{1 / 2}+R \cdot N_{1 / 2}(u)
$$

La figure 5 présente les interférences qu'engendre un positionnement SPO, celles-ci sont de type overcut au niveau des deux directrices.

Avantages :

- le calcul du positionnement outil est simple;

- l'erreur d'interférence est bien réduite par rapport au positionnement logiciel.

Inconvénient :

- l'erreur d'interférence n'est que de l'overcut. 


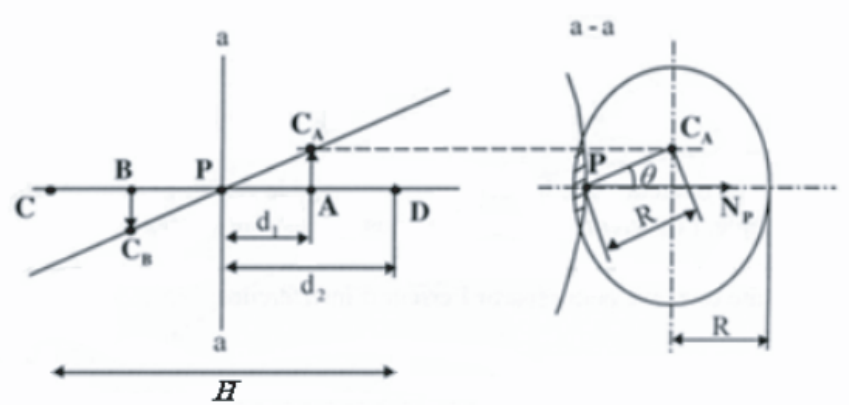

Fig. 6. Posage de l'outil en positionnement DPO [5].

\section{Positionnement standard}

Le positionnement standard permet de répartir équitablement les interférences sur les deux directrices. L'axe de l'outil est donc toujours colinéaire à la règle et une génératrice de l'outil est confondue avec la règle [6].

Il faut donc calculer le point de tangence sur la règle pour avoir la même interférence sur les deux directrices. Ce problème n'est pas facile à résoudre, ce qui va impliquer un temps de calcul long pour le posage de l'outil. Il est important de souligner ce problème même si aujourd'hui les moyens de calculs sont performants, car ce calcul doit être exécuté pour chaque point de discrétisation de la trajectoire outil.

Ce posage permet donc de répartir les interférences, qui restent de type overcut, équitablement sur les deux directrices et ainsi en réduire la valeur maximale.

Avantages :

- l'erreur d'interférence est bien diminuée par rapport au positionnement logiciel;

- l'erreur d'interférence est répartie entre les deux directrices.

Inconvénients :

- le calcul du posage outil est complexe ce qui rend difficile son implantation dans les logiciels de FAO;

- l'erreur d'interférence n'est que de l'overcut.

\subsection{Posage avec deux points de tangence}

\section{Positionnement DPO}

Le second posage proposé par Liu [5] est un posage bitangent nommé Double Point Offset (DPO). L'outil est ici tangent à deux points de la surface situés à $1 / 4$ et $3 / 4$ de la règle qui sont respectivement $\mathrm{A}$ et $\mathrm{B}$ sur la figure 6 . Deux points de passage de l'axe outil, respectivement $C_{\mathrm{A}}$ et $C_{\mathrm{B}}$ sont déterminés pas les équations $(6),(7)$ :

$$
\begin{aligned}
& C_{\mathrm{A}}=A+R \boldsymbol{N}_{\mathrm{A}} \\
& C_{\mathrm{B}}=B+R \boldsymbol{N}_{\mathrm{B}}
\end{aligned}
$$

$\boldsymbol{N}_{\mathrm{A}}$ et $\boldsymbol{N}_{\mathrm{B}}$ étant les normales à la surface respectivement aux points $\mathrm{A}$ et $\mathrm{B}$. Le point milieu de la règle sera noté $\mathrm{P}$ avec $N_{\mathrm{P}}$ la normale en ce point. La figure 6 permet de montrer que l'interférence ainsi obtenue se répartit en

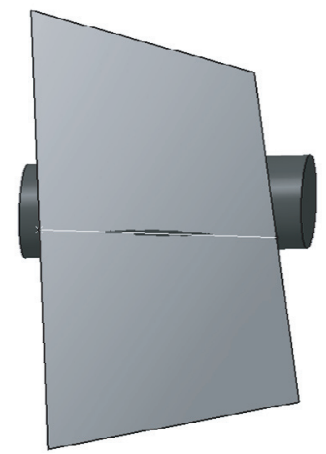

Fig. 7. Interférences obtenues avec le positionnement DPO.

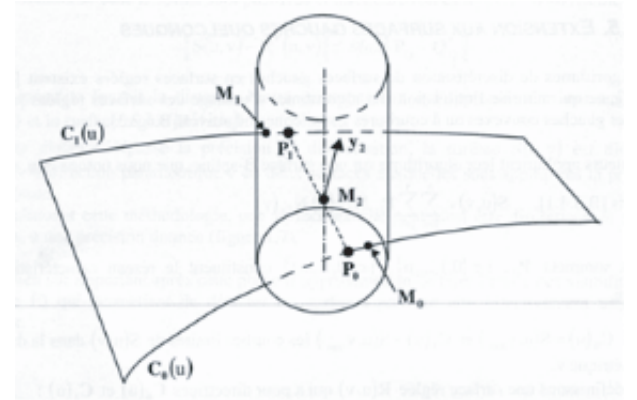

Fig. 8. Posage de l'outil avec le positionnement amélioré [7].

overcut entre les deux points de tangence, maximale en $\mathrm{P}$, et en undercut à l'extérieur, maximale en $\mathrm{C}$ et $\mathrm{D}$.

Avantages :

- l'erreur d'interférence est bien réduite par rapport au positionnement logiciel;

- l'erreur d'interférence est répartie en overcut et en undercut comme l'illustre la figure 7 ;

- le temps du calcul est rapide et peut être résolu algébriquement.

Inconvénient :

- le choix des points de tangence est arbitraire.

\subsection{Posage avec trois points de tangence}

\section{Positionnement amélioré}

Ce positionnement mis au point par Redonnet [7] est une amélioration du positionnement standard. Ce positionnement part d'un outil en position standard et lui fait subir une rotation autour de $y_{2}$ de telle sorte que l'outil soit en triple tangence en M0, M1 et M2. La figure 8 présente l'outil en positionnement amélioré. Le positionnement de l'outil est optimisé en résolvant numériquement un système non-linéaire de sept équations à sept inconnues. Ce positionnement crée deux zones d'overcut de part et d'autre du point M2.

Avantages :

- l'erreur d'interférence est bien réduite par rapport au positionnement logiciel. Pour donner un ordre de grandeur, l'erreur est divisée en moyenne par un facteur cinq par rapport au positionnement standard. 


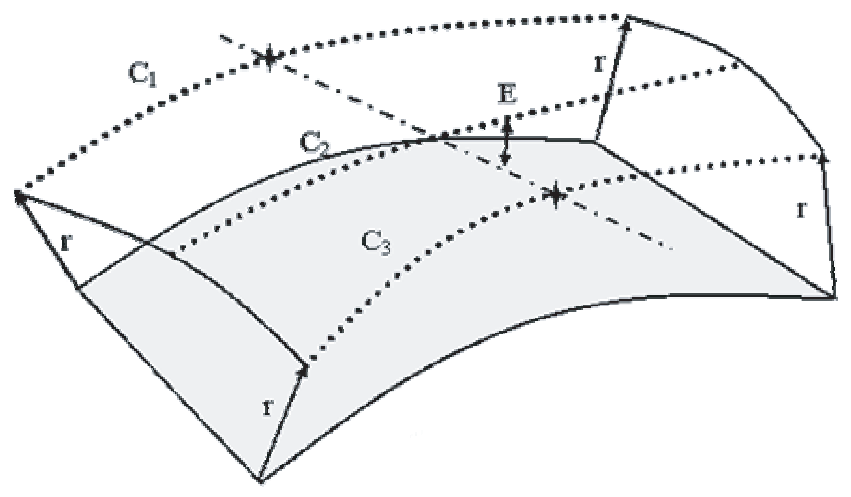

Fig. 9. Positionnement TPO.

Inconvénients :

- le temps du calcul est long et ne peut pas être résolu algébriquement;

- l'erreur d'interférence est uniquement de l'overcut;

- la détermination algébrique du rayon d'outil maximal n'est pas possible.

\section{Positionnement TPO (Three Points Offset)}

Ce positionnement mis au point par H. Gong [8] se détache des précédents par le fait qu'il positionne l'axe de l'outil sur la surface offset et non l'outil sur la surface à usiner. L'idée de base de ce positionnement est que la surface enveloppe des axes outils, lors de l'usinage flanc, est une SRND. Or la surface offset d'une SRND est une surface qui n'est pas réglée (car du fait que la normale évolue le long d'une règle, l'offset d'une règle devient une courbe et non une droite). Cette méthode permet donc de poser l'axe outil sur une surface qui n'est pas réglée.

Pour positionner l'axe outil, les étapes sont les suivantes (voir Fig. 9):

- construction de la surface offset, de la valeur du rayon de l'outil qui va l'usiner;

- les courbes offset des iso-paramétriques à $0,0,5$ et 1 sont nommées respectivement $C_{1}, C_{2}$ et $C_{3}$;

- l'axe de l'outil passe par $C_{1}$ et $C_{3}$ puis la distance $E$, entre l'axe outil et $C_{2}$, est minimisé;

- finalement l'axe outil coupe les trois courbes offset, d'où le nom TPO.

Avantages :

- l'erreur d'interférence est bien réduite par rapport au positionnement logiciel;

- les directrices ont très peu d'interférence.

Inconvénient :

- le temps du calcul est long et ne peut pas être résolu algébriquement.

\subsection{Positionnement global sur la surface}

\section{Positionnement LURPA}

Ce positionnement mis au point au LURPA [9] est totalement différent des autres car il ne pose pas l'outil sur plusieurs points de la surface mais il pose une surface usinée au global sur une surface nominale. Pour cela il faut suivre les étapes suivantes :

- positionner l'outil avec un posage simple (un présenté précédemment par exemple) tout en sachant que l'on va obtenir de l'interférence;

- évaluation de la surface enveloppe, ce concept est le point de base de cette méthode. La surface enveloppe est la surface théorique que l'outil génère lorsqu'il suit une trajectoire d'usinage;

- discrétisation de la surface à usiner. Calcul de l'écart entre les points obtenus par discrétisation et la surface enveloppe;

- modification de la surface enveloppe sous contrainte de réduire les écarts précédemment calculés.

Avantages :

- l'erreur d'interférence est réduite par rapport au positionnement logiciel ;

- la trajectoire obtenue est lisse du fait qu'elle est définie mathématiquement par une seule surface.

Inconvénient :

- le temps du calcul est long.

La méthodologie de développement des fraises décrite dans le chapitre suivant sera développée dans le cadre du positionnement SPO.

\section{Usinage de SRND avec des fraises tonneaux}

Il ressort de l'état de l'art précédemment réalisé que l'usinage de SRND est encore de nos jours un problème. Les différentes solutions apportées par la bibliographie utilisent principalement comme degré de liberté la trajectoire. Mais il y a un autre degré de liberté qui est à l'heure actuelle sous-exploité; c'est la forme de l'outil. En effet pour l'instant les seuls degrés de liberté utilisés sur la forme de l'outil sont :

- le type d'outil : cylindrique ou conique;

- le diamètre de l'outil qui influe directement sur l'interférence générée.

Les travaux présentés ici s'intéressent à optimiser la forme de l'outil pour réduire l'interférence générée.

Les données d'entrées du calcul des fraises tonneaux sont la surface et le rayon nominal de l'outil tonneau. La figure 10 présente un outil tonneau, défini par deux rayons :

- $r$ : rayon maximal de l'outil;

- $R$ : le rayon du tonneau.

\subsection{Principe des fraises tonneaux mono-passe}

La figure 11 présente le principe de base des fraises tonneaux; cette idée part de l'observation de la forme de l'interférence obtenue lors du posage d'un outil cylindrique sur une SRND en positionnement SPO. Cette interférence est de l'overcut de part et d'autre du point de tangence comme le montre la figure 11 . Il semble donc naturel de chercher à réduire le diamètre de l'outil dans ces 


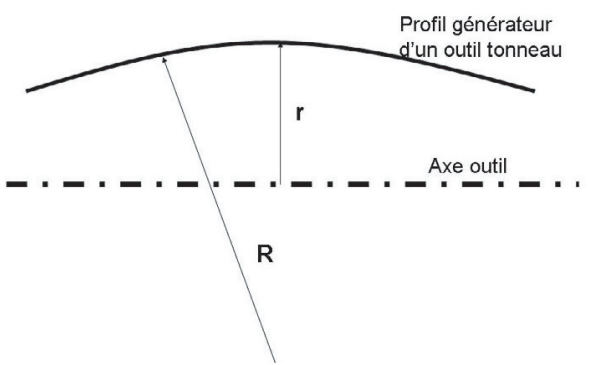

Fig. 10. Définition d'un outil tonneau.

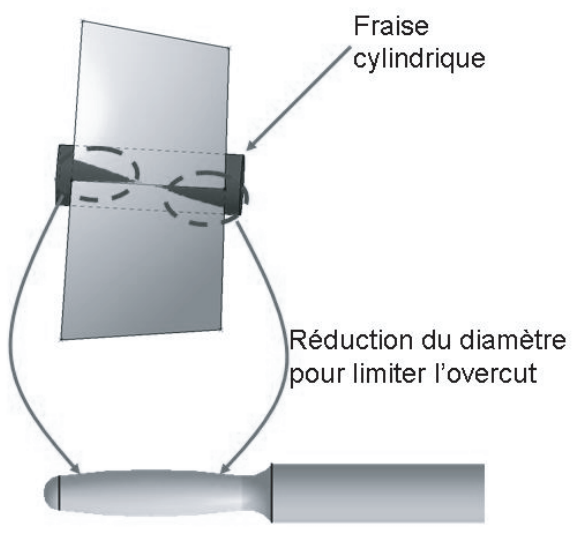

Fig. 11. Principe des fraises tonneaux.

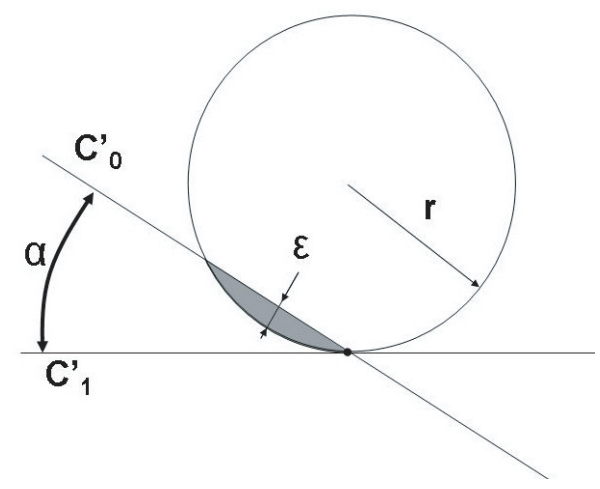

Fig. 12. Calcul de l'interférence.

zones là. Ces réductions du diamètre de l'outil lui donnent une forme de tonneau, d'où son nom.

\subsection{Calculs préliminaires}

Pour estimer la diminution de diamètre nécessaire pour limiter l'interférence, il faut savoir calculer cette valeur d'interférence pour un outil cylindrique. Pour simplifier les calculs, l'interférence entre la surface et l'outil sera estimée par l'interférence entre la tangente à la surface, projetée sur un plan perpendiculaire à la règle $C_{0}^{\prime}$ et $C_{1}^{\prime}$ et l'outil comme le montre la figure 12 .

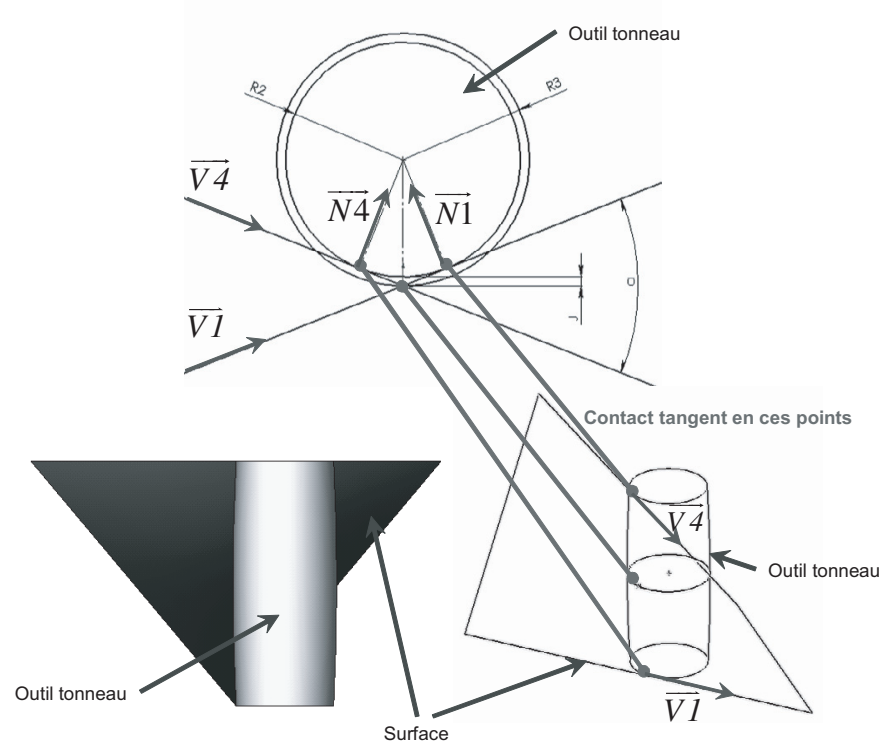

Fig. 13. Méthodologie de calcul du profil de tonneau.

Cette valeur d'interférence notée $\varepsilon$, est obtenue grâce à l'équation (8):

$$
\varepsilon=r \cdot(1-\cos (\alpha))
$$

\subsection{Calcul du profil de fraise tonneau}

Pour chaque isoparamétrique $u$, un profil d'outil est calculé pour usiner avec le moins possible d'interférences. Le profil tonneau sera calculé en utilisant les contraintes suivantes :

- Contact tangent entre l'outil tonneau et la surface aux points de paramètre $v=0, v=0.5$ et $v=1$. Ceci permet d'usiner sans interférences les deux directrices et l'isoparamétrique à $v=0,5$.

- Le diamètre nominal de l'outil, $r$, sera le plus grand diamètre du tonneau, il sera donné au logiciel en fonction de l'espace disponible pour usiner la surface. Ce diamètre devra, lors de la programmation FAO, suivre l'isoparamétrique à $v=0,5$.

Ces contraintes sont illustrées sur la figure 13 .

De plus pour réaliser le calcul du rayon du tonneau, $R$, l'hypothèse est faite que la vrille varie linéairement le long d'une règle. Avec cette hypothèse, l'angle formé entre $\boldsymbol{N}_{0}$ et $\boldsymbol{N}_{1 / 2}$ et l'angle formé entre $\boldsymbol{N}_{1 / 2}$ et $\boldsymbol{N}_{1}$ sont connus et ils sont tous les deux égaux à $\alpha / 2$. Le calcul obtenu préalablement donne l'interférence générée en $P_{0}$ et $P_{1}$ (voir la Fig. 1 pour les notations).

Avec toutes ces informations, le calcul du rayon de tonneau peut être réalisé. Toutes les informations sont synthétisées sur la figure 14 qui représente le profil générateur de l'outil tonneau pour usiner une position donnée. 


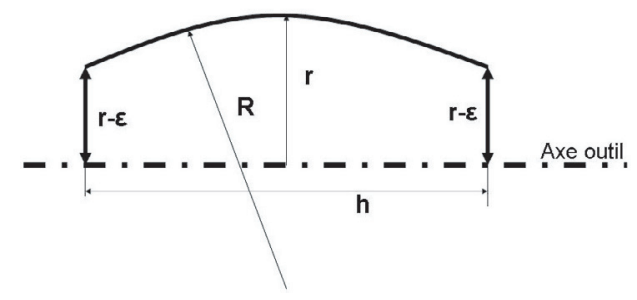

Fig. 14. Donnée pour le calcul de $R$.

Ces informations permettent d'obtenir l'équation (9) :

$$
R(u)=-\frac{(h(u) / 2)^{2}+(r \cdot \cos (\alpha(u) / 2)-r)^{2}}{2 \cdot(r \cdot \cos (\alpha(u) / 2)-r)}
$$

Ce calcul nous fournit la valeur de $R(u)$ pour un point de la trajectoire. Mais pour réaliser une surface il faut avoir une seule fraise. Le choix est fait de prendre un outil global avec $R$ étant la moyenne des $R(u)$ d'où l'équation (10) :

$$
R=\text { moyenne }(R(u))
$$

Et une longueur utile de l'outil, $L_{\text {utile, plus grande }}$ que la plus longue des règles ce qui se traduit par l'équation (11)

$$
L_{\text {utile }}=\max (h)
$$

L'équation (9) permet de montrer que la forme de tonneau dépend de la vrille et de la longueur des règles. Ceci explique la définition des deux indicateurs mentionnés précédemment, qui s'intéressent à la variation de ces deux grandeurs.

\section{Conclusions}

Actuellement, les fraises tonneau sont très peu utilisées industriellement. Elles sont principalement utilisées pour le fraisage de forme afin de reproduire un profil en forme de tonneau sur la pièce.

Cependant, cette étude a montré que ces outils permettent aussi de réduire les interférences locales générées lors de l'usinage d'une SRND. Les stratégies fraise tonneau mono-passe (la surface est balayée en une seule fois) et passes multiples (la surface est balayée en plusieurs passes) permettent un gain de temps important par rapport à la stratégie d'usinage en bout avec une fraise hémisphérique.

Le logiciel COAO.xls développé pendant ce projet calcule automatiquement la forme de l'outil tonneau le mieux adapté à la surface de façon automatique.

Les principaux inconvénients des stratégies fraise tonneau avec un positionnement SPO sont :

- un calcul de la fraise en moyenne sur toute la surface ce qui induit une moins bonne efficacité sur les surfaces qui varient beaucoup. Pour quantifier cette variation deux indicateurs ont été mis en place;

- un outil dédié à une surface.

Pour pallier ce dernier problème la surface peut être usinée avec une stratégie tonneau multi-passes qui permet d'avoir un outil avec un rayon de tonneau plus standard et qui reste quand même plus économique que l'usinage en bout.

\section{Références}

[1] Y. Takeuchi, K. Morishige, M. Yokoyama, T. Hisaki, 5axis control machining using side-milling cutter, J. Adv. Automation Tech. 7 (1995) 60-65

[2] F. Rehsteiner, Collision free five-axis milling of twisted ruled surfaces, Annals of the CIRP 42 (1993) 457-461

[3] D. Vouillon, De la modélisation à l'usinage 5 axes d'un impeller, Mémoire CNAM ENSAM de Cluny 2003.

[4] H. Tönshoff, C. Gey, N. Rackow, Flank milling optimization, the flamingo project, Air \& Space Europe, 3 (2001) 60-63

[5] X.-W. Liu, Five-axis NC cylindrical milling of sculptured surfaces, Computer Aided Design 27 (1995) 887-894

[6] W. Rubio, P. Lagarrigue, G. Dessein, F. Pastor, Calculation of tool paths for a torus mill on free-form surfaces five-axis machines with detection and elimination of interference, Int. J. Adv. Manufacturing Tech. 14 (1998) $13-20$

[7] J.M. Redonnet, W. Rubio, G. Dessein, Side milling of ruled surfaces - optimum positioning of the milling cutter and calculation of interference, Int. J. Adv. Manufacturing Tech. 14 (1998) 459-465

[8] Hu Gong, Li-Xin Cao, Jian Liu, Improved positioning of cylindrical cutter for flank milling ruled surfaces, Computer-Aided Design 37 (2005) 1205-1213

[9] C. Lartigue, E. Duc, A. Affouard, Tool path deformation in 5-axis flank milling using envelope surface, Computer Aided Design 35 (2003) 375-382 